\title{
Microtubule-Associated Protein 1A (MAP1A) and MAP1B: Light Chains Determine Distinct Functional Properties
}

\author{
Rainer Noiges, René Eichinger, Waltraud Kutschera, Irmgard Fischer, Zsuzsanna Németh, Gerhard Wiche, \\ and Friedrich Propst
}

Institute of Biochemistry and Molecular Cell Biology, Vienna Biocenter, University of Vienna, A-1030 Vienna, Austria

\begin{abstract}
The microtubule-associated proteins $1 \mathrm{~A}(\mathrm{MAP} 1 \mathrm{~A})$ and 1B (MAP1B) are distantly related protein complexes consisting of heavy and light chains and are thought to play a role in regulating the neuronal cytoskeleton, MAP1B during neuritogenesis and MAP1A in mature neurons. To elucidate functional differences between MAP1B and MAP1A and to determine the role of the light chain in the MAP1A protein complex, we chose to investigate the functional properties of the light chain of MAP1A (LC2) and compare them with the light chain of MAP1B (LC1). We found that LC2 binds to microtubules in vivo and in vitro and induces rapid polymerization of tubulin. A microtubule-binding domain in its $\mathrm{NH}_{2}$ terminus was found to be necessary and sufficient for these activities. The analysis of LC1 revealed that it too bound to microtubules and induced tubulin polymeriza-
\end{abstract}

The high-molecular-mass microtubule-associated proteins $1 \mathrm{~A}$ (MAP1A) and 1B (MAP1B) are expressed predominantly in cells of the nervous system (Wiche et al., 1991; Schoenfeld and Obar, 1994). Expression of MAP1B is high during early stages of neuronal development and is downregulated in the adult (Binder et al., 1984; Calvert and Anderton, 1985; Lewis et al., 1986; Riederer et al., 1986; Safaei and Fischer, 1989; Schoenfeld et al., 1989; Garner et al., 1990). MAP1A exhibits a reciprocal pattern of expression, reaching its peak in the adult brain, when neuronal differentiation is complete (Schoenfeld et al., 1989; Garner et al., 1990). MAP1B has been shown to play an important role in neuronal differentiation in vitro (Brugg et al., 1993; DiTella et al., 1996; Gonzalez-Billault et al., 2001) and in the development of the murine nervous system in vivo (Edelmann et al., 1996; Takei et al., 1997; González-Billault et al., 2000; Meixner et al., 2000). There are no comparable data for MAP1A.

Both MAP1A and MAP1B are multimeric protein complexes containing one heavy and several light chains; they share isolated domains of sequence homology in their subunits (Hammarback et al., 1991; Langkopf et al., 1992). In each case, the heavy chain and one of the light chains are generated by proteolytic cleavage of the respective MAP1A or MAP1B polyprotein precursor. These subunits are interchangeable (Schoenfeld et al., 1989). Thus, the MAP1B light chain, LC1, can bind to both MAP1A and MAP1B heavy chains. The same is true for the MAP1A light chain, LC2,

Received Aug. 13, 2001; revised Nov. 20, 2001; accepted Dec. 3, 2001.

This research was supported by a grant from the Austrian Science Fund (Project No. F607). We thank Albert Weixelbaumer for his contribution in the biochemical analyses.

Correspondence should be addressed to F. Propst, Institute of Biochemistry and Molecular Cell Biology, Dr. Bohr-Gasse 9, A-1030 Vienna, Austria. E-mail: friedrich.propst@univie.ac.at.

Copyright (C) 2002 Society for Neuroscience $0270-6474 / 02 / 222106-09 \$ 15.00 / 0$ tion via a crucial but structurally unrelated $\mathrm{NH}_{2}$-terminal domain. The two light chains differed, however, in their effects on microtubule bundling and stability in vivo. Furthermore, we identified actin filament binding domains located at the $\mathrm{COOH}$ terminus of LC2 and LC1 and obtained evidence that binding to actin filaments is attributable to direct interaction with actin. Our findings establish LC2 as a crucial determinant of MAP1A function, reveal LC2 as a potential linker of neuronal microtubules and microfilaments, and suggest that the postnatal substitution of MAP1B by MAP1A leads to expression of a protein with an overlapping but distinct set of functions.

Key words: actin; brain development; microtubule-associated proteins; microtubule stability; neuronal cytoskeleton; tubulin polymerization although it binds predominantly to the heavy chain of MAP1A. Moreover, there is evidence that the light chains might have additional functions outside of the complex with the heavy chains. It was found that $\mathrm{LC} 1$ is expressed at levels in excess over what can be complexed by heavy chains (Mei et al., 2000b).

MAP1A and MAP1B bind to microtubules (Schoenfeld and Obar, 1994) and microfilaments (Asai et al., 1985; Pedrotti et al., 1994; Pedrotti and Islam, 1996; Tögel et al., 1998b), suggesting that they are involved in mediating or regulating the interaction between axonal microtubules and actin filaments, which is believed to be essential for neuronal morphogenesis and function. Association of MAP1B with microtubules is mediated by two unique microtubule-binding domains, located on the heavy chain (Noble et al., 1989) and light chain (Zauner et al., 1992; Tögel et al., 1998b), respectively. For MAP1A, two unrelated regions, both located in the heavy chain, have been implicated in microtubule binding (Cravchik et al., 1994; Vaillant et al., 1998). The role of the light chain for MAP1A interaction with microtubules and microfilaments has not been determined, and it is not clear whether MAP1A and MAP1B interact directly with actin or actin-binding proteins. To clarify these questions and to determine the contribution of the light chains to MAP1A and MAP1B function, we analyzed properties of the light chains by biochemical and cell biological techniques in vivo and in vitro.

\section{MATERIALS AND METHODS}

cDNA constructs. LC 2 cDNA was obtained by reverse transcription PCR using the primers LC2U (5'-CGGAGTCGACCATG GCTGACCCTGAGGGG-3') and LC2L (5'-CGCATCTAGAGCTAGCGTGAACTCAATCTTGCAGGC $-3^{\prime}$ ). The correct sequence of this cDNA was confirmed. It encodes amino acids 2554-2774 of rat MAP1A (Langkopf et al., 1992). Constructs encoding full-length LC2 (amino acids 25542774), its $\mathrm{NH}_{2}$ terminus (amino acids 2554-2659), or its $\mathrm{COOH}$ terminus (amino acids 2650-2774) all fused in frame to an $\mathrm{NH}_{2}$ - or $\mathrm{COOH}$ - 
terminal myc-tag [amino acid sequence: EQKLISEEDLN (Cravchik and Matus, 1993)] by a linker of three amino acids were generated using convenient restriction sites and adapter oligonucleotides. Constructs encoding full-length $\mathrm{LC} 1$ and its $\mathrm{NH}_{2}$ - and $\mathrm{COOH}$-terminal domains have been described previously (Tögel et al., 1998b). All constructs were cloned into the mammalian Tet-Off expression vector pUHD10-3 (Gossen and Bujard, 1992) and into a pET15b (Novagen, Inc., Madison, WI) or pQE60 (Qiagen, Hilden, Germany) derivative for the expression of $\mathrm{NH}_{2}-$ or $\mathrm{COOH}$-terminal 6xHis-tagged proteins in Escherichia coli. The authenticity of all constructs was confirmed by sequencing and/or reaction of encoded proteins with LC1- or LC2-specific antisera.

Antibodies. A rabbit polyclonal anti-LC2 antiserum was raised against the synthetic peptide CKGPVDRTSRTVPRPR (MAP1A amino acids 2605-2619; Gramsch, Schwabhausen, Germany). The anti-LC1 antiserum has been described previously (Tögel et al., 1998b). An affinitypurified polyclonal rabbit anti-myc antibody (Tögel et al., 1998a) was used at a concentration of $1 \mu \mathrm{g} / \mathrm{ml}$ for immunofluorescence microscopy. Intracellular microtubules were detected with mouse anti-tubulin monoclonal antibody B-5-1-2 (Sigma, St. Louis, MO) at a dilution of 1:500, and actin stress fibers were detected using a mixture of mouse anti- $\beta$-actin monoclonal antibodies AC-15 and AC-74 (Sigma) at a dilution of 1:200. Texas Red-labeled anti-mouse and FITC-labeled anti-rabbit secondary antibodies (Jackson ImmunoResearch Laboratories, Inc., West Grove, PA) were used at dilutions of 1:100.

Cell culture, DNA transfection, and immunofluorescence microscopy. PtK2 cells were grown at $37^{\circ} \mathrm{C}$ in an atmosphere containing $8.5 \% \mathrm{CO}_{2}$ in high glucose DMEM supplemented with $10 \%$ FCS in the absence of tetracycline. At $18-20 \mathrm{hr}$ before transfection, cells were seeded onto coverslips at a density of $66 \%$. Transient transfection was performed using Lipofectamine (Invitrogen, Bethesda, MD) according to the manufacturer's protocol. The ratio of expression plasmid to transactivator plasmid was 10:1. The transfection mixture was replaced with fresh growth medium after $5 \mathrm{hr}$, and the cells were incubated for an additional $48 \mathrm{hr}$ at $37^{\circ} \mathrm{C}$.

For immunofluorescence microscopy, PtK2 cells were washed in PBS, fixed in methanol $\left(-20^{\circ} \mathrm{C}, 10 \mathrm{~min}\right)$, equilibrated in PBS, blocked for 30 min with $3 \%$ BSA, incubated with the primary antibodies in 1\% BSA for $1 \mathrm{hr}$, washed extensively in PBS, incubated for $1 \mathrm{hr}$ with the secondary antibodies, and washed again with PBS. Specimens were analyzed by confocal microscopy using a Zeiss Axiovert microscope (Carl Zeiss, Oberkochen, Germany).

For quantitative analysis, experiments were chosen in which expression of both constructs was equal as judged by immunofluorescence intensity. The presence of stable microtubules was assessed in 100 randomly chosen transfected cells in at least two independent experiments.

Purification of recombinant proteins. Recombinant proteins were expressed in E. coli BL21-CodonPlus-RIL or E. coli XL1-Blue and purified by affinity chromatography on $\mathrm{Ni}^{2+}$ columns, according to the manufacturers' protocols (Novagen, Inc.; Qiagen). Recombinant proteins were bound to and eluted from the column in the presence of $6 \mathrm{M}$ urea, except for the $\mathrm{LC} 1 \mathrm{NH}_{2}$ terminus, which was purified in the absence of urea. Before biochemical analysis, recombinant proteins were extensively dialyzed at $4^{\circ} \mathrm{C}$ against buffers used in the respective in vitro assays. Protein concentrations were determined according to the method of Bradford (1976) using BSA as a standard.

Purification of tubulin. Tubulin devoid of microtubule-associated proteins was prepared as described previously (Karr et al., 1979), with the following modifications. Polymerization of tubulin was performed in PEM buffer (PIPES $0.1 \mathrm{M}$, EGTA $2 \mathrm{~mm}, \mathrm{MgCl}_{2} 1 \mathrm{~mm}, \mathrm{pH}$ 6.8) containing $0.1 \mathrm{~mm}$ GTP. Centrifugation steps were performed at $100,000 \times g$ in a Beckman TLX tabletop ultracentrifuge (Beckman Instruments, Inc., Palo Alto, CA). The last cycle of polymerization/depolymerization was followed by chromatography on a phosphocellulose column, as described previously (Weinert et al., 1982). After precipitation, the protein was resuspended in PEM buffer. Protein purity was monitored using SDSPAGE and Coomassie blue staining.

Microtubule cosedimentation. Cosedimentation assays were performed as described previously (Feick et al., 1991), with slight modifications. In the presence of $5 \mathrm{~nm}$ taxol, tubulin was polymerized for $30 \mathrm{~min}$ at $37^{\circ} \mathrm{C}$. LC1 and LC2 proteins were dialyzed against $0.1 \mathrm{M}$ PEM buffer and centrifuged for $30 \mathrm{~min}$ at $100,000 \times g$ to remove any aggregates. Then 50 $\mu \mathrm{g}$ of polymerized tubulin was incubated with $10 \mu \mathrm{g}$ of LC1 or LC2 proteins for $10 \mathrm{~min}$ at $37^{\circ} \mathrm{C}$. Samples were layered onto a $10 \%$ sucrose cushion and centrifuged for $20 \mathrm{~min}$ at $10,000 \mathrm{rpm}$ in a tabletop centri- fuge. Equal volumes of supernatants and pellets were analyzed by SDSPAGE and Coomassie blue staining.

Turbidity assay. Polymerization of tubulin was performed as described previously (Weinert et al., 1982), with the following modifications. Proteins dialyzed against $0.1 \mathrm{M}$ PEM buffer were mixed with $1 \mathrm{~mm}$ GTP and $1.5 \mathrm{mg} / \mathrm{ml}$ tubulin. Polymerization was initiated by placing the mixture immediately into $37^{\circ} \mathrm{C}$ prewarmed $0.25 \mathrm{ml}$ cuvettes, and turbidity change was followed at $350 \mathrm{~nm}$ using a Hitachi (Tokyo, Japan) U-3000 spectrophotometer equipped with a thermostat.

Europium overlay binding assay. Binding assays were performed as described previously (Steinböck et al., 2000). Briefly, LC1 and LC2 proteins were dialyzed against $\mathrm{Eu}^{3+}$-labeling buffer. Ninety-six well microtiter plates were coated with $100 \mathrm{~nm}$ actin (rabbit skeletal muscle actin; Cytoskeleton, Inc., Denver, CO) or BSA type H1 (Gerbu, Gaiberg, Germany) as a control. After blocking with 4\% BSA, plates were overlaid with increasing amounts of $\mathrm{Eu}^{3+}$-labeled LC1 or LC2 proteins. Plates were washed, and bound protein was determined by releasing the complexed $\mathrm{Eu}^{3+}$ with enhancement solution and measuring fluorescence with a Delfia time-resolved fluorometer (Wallac, Turku, Finland). Binding of LC1 or LC2 proteins to BSA was considered to be nonspecific and was subtracted from protein bound to actin. Counts were converted to concentrations by comparison with a $1 \mathrm{nM} \mathrm{Eu}^{3+}$ standard.

Actin cosedimentation. $\mathrm{LC} 1$ and $\mathrm{LC} 2$ proteins were dialyzed against 0.1 M MES buffer and centrifuged for $30 \mathrm{~min}$ at $100,000 \times g$ to remove any aggregates. Actin (rabbit skeletal muscle actin; Cytoskeleton, Inc.) was polymerized (Pedrotti and Islam, 1996) and incubated with $3 \mu \mathrm{g}$ of recombinant LC1 or LC2 protein. Samples were centrif uged at 100,000 $\times$ $g$ for $30 \mathrm{~min}$ at $37^{\circ} \mathrm{C}$, and equal volumes of supernatants and pellets were analyzed by SDS-PAGE and Coomassie blue staining.

\section{RESULTS}

\section{The MAP1A light chain binds and stabilizes microtubules in vivo}

Microtubule association of the MAP1A complex has thus far been attributed to microtubule-binding domains located in the heavy chain (Cravchik et al., 1994; Vaillant et al., 1998). We decided to investigate the potential involvement of the MAP1A light chain in microtubule binding. We used PtK2 cells, which have been shown previously to express low levels of LC1 (Tögel et al., 1998b) that faintly decorated their microtubules (data not shown). These cells also expressed low levels of LC2 in the cytoplasm and the nucleus (data not shown). PtK2 cells were transiently transfected with a cDNA construct encoding the fulllength LC2 protein and analyzed by double immunofluorescence microscopy. In cells expressing LC2, the myc-tagged protein was found to colocalize with microtubules, which were decorated by LC2 in a punctate manner (Fig. $1 A, B$ ). The merge of the confocal images obtained with anti-myc and anti-tubulin antibodies revealed that not all microtubules were decorated to the same extent. The decoration was more pronounced toward the perinuclear region (Fig. 1C). Whereas expression of $\mathrm{LC} 1$ induces severe changes in microtubule organization (Tögel et al., 1998b), binding of LC2 to microtubules did not affect their appearance and organization (Fig. 1, compare $B$ and $E$ ). Microtubules in LC2expressing cells were indistinguishable from those in untransfected cells (data not shown). In contrast, many microtubules in LC1-expressing cells were organized into thick, wavy bundles, some of which are pointed out by arrows (Fig. $1 E$ ). Staining with the anti-myc antibody also revealed nuclear localization of LC2 (Fig. $1 A$ ). This was also observed for endogenous LC2 in untransfected cells (data not shown) and in LC2-transfected NIH3T3 cells (Mei et al., 2000a). Microtubule association of LC1 and LC2 is unlikely to be affected by the presence of the myc-tag, because untagged LC1 yielded identical results (data not shown). Neither LC1 nor LC2 overexpression in PtK2 cells induced gross morphological changes.

On the basis of these findings, we further analyzed whether 

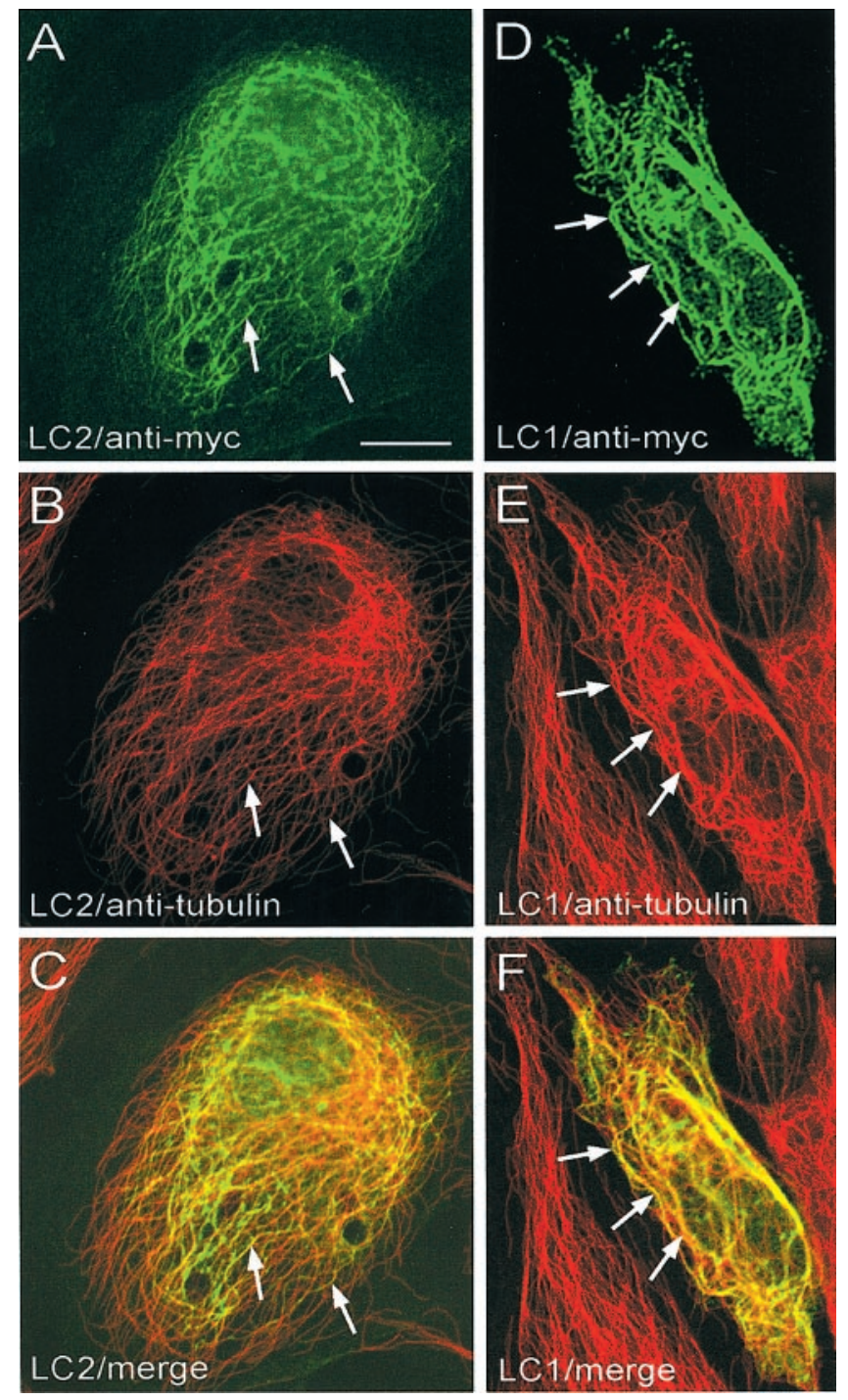

Figure 1. The LC2 protein binds to microtubules in vivo. Shown are confocal images of PtK2 cells expressing myc-tagged LC2 $(A-C)$ or myc-tagged LC1 $(D-F)$, analyzed by double immunofluorescence microscopy using antibodies against the myc-tag $(A, D)$ and tubulin $(B, E)$. LC2 and LC1 colocalized with microtubules (arrows), but LC2 did not induce formation of wavy microtubule bundles like LC1 (compare $B$ and $E$ ). $C$ and $F$ represent merged confocal images of $A+B$ and $D+E$, respectively. Scale bar, $10 \mu \mathrm{m}$.

expression of LC2 altered microtubule stability. Treatment of cells with the microtubule depolymerizing agents colchicine and nocodazole before fixation led to destruction of the cellular microtubule network, resulting in a diffuse distribution of tubulin throughout the cytoplasm (Figs. 2D, 3D). In cells expressing LC2, we observed integrity of the cellular microtubules despite treatment with colchicine (Fig. 2A,B). The merge of the confocal images obtained with anti-myc and anti-tubulin antibodies revealed that stable microtubules were decorated with LC2 (Fig. $2 C$ ). This is consistent with the assumption that binding of LC2 is necessary for stabilization. The same was observed for cells expressing LC1 (Fig. 2E-G). Quantitative analysis of these experiments and comparison with $\mathrm{LC} 1$ revealed that both proteins, LC1 and LC2, were equally efficient in protecting cellular microtubules against the depolymerizing effects of colchicine (Fig. $2 H$ ). In cells expressing LC1 or LC2, intact microtubules were ob-
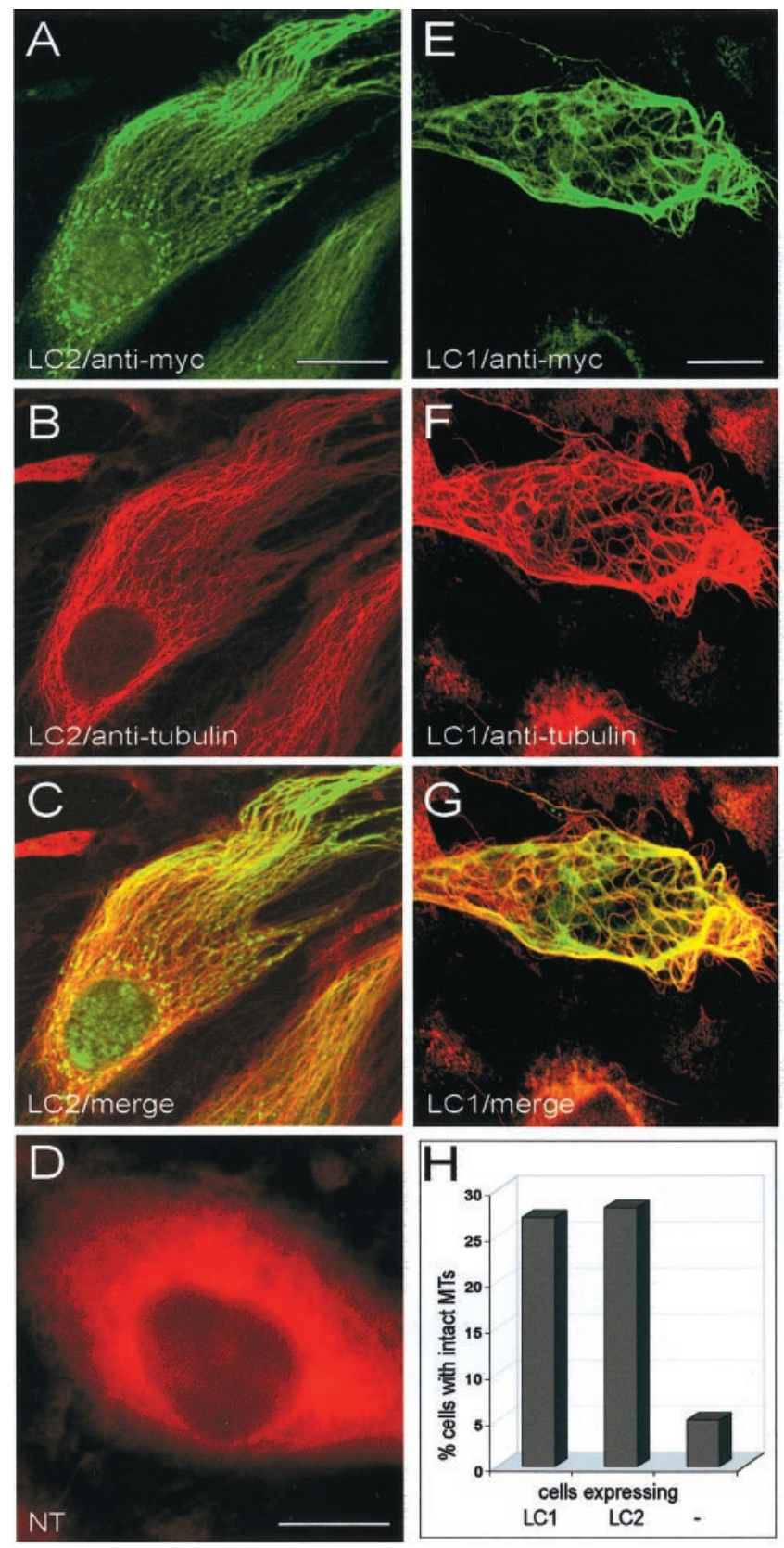

Figure 2. Effects of LC2 and LC1 on microtubule stability in cells treated with colchicine. Nontransfected PtK2 cells $(N T, D)$ or PtK2 cells transfected with myc-tagged LC2 $(A-C)$ or myc-tagged LC1 $(E-G)$ were treated with colchicine $(1-2 \mathrm{hr}, 10 \mu \mathrm{M})$ and then analyzed by double immunofluorescence microscopy using antibodies against tubulin $(B, D$, $F)$ and the myc-tag $(A, E)$. Microtubules were depolymerized in nontransfected cells $(D)$, whereas intact microtubules were found in cells expressing LC2 $(B)$ and LC1 $(F) . C$ and $G$ represent merged confocal images of $A+B$ and $E+F$, respectively. Scale bars, $20 \mu \mathrm{m}$. $H$, One hundred randomly chosen transfected cells were assessed for the presence of intact microtubules (MTs). Quantitative analysis revealed that LC1 and LC2 were equally efficient in protecting microtubules against depolymerization (-, untransfected cells).

served in a subpopulation of 27 and $28 \%$, respectively, despite colchicine treatment.

In contrast, in cells treated with nocodazole, the protective effect of LC1 was much more pronounced than that of LC2 (Fig. 3 ). With either light chain, cells with stable microtubules could be 

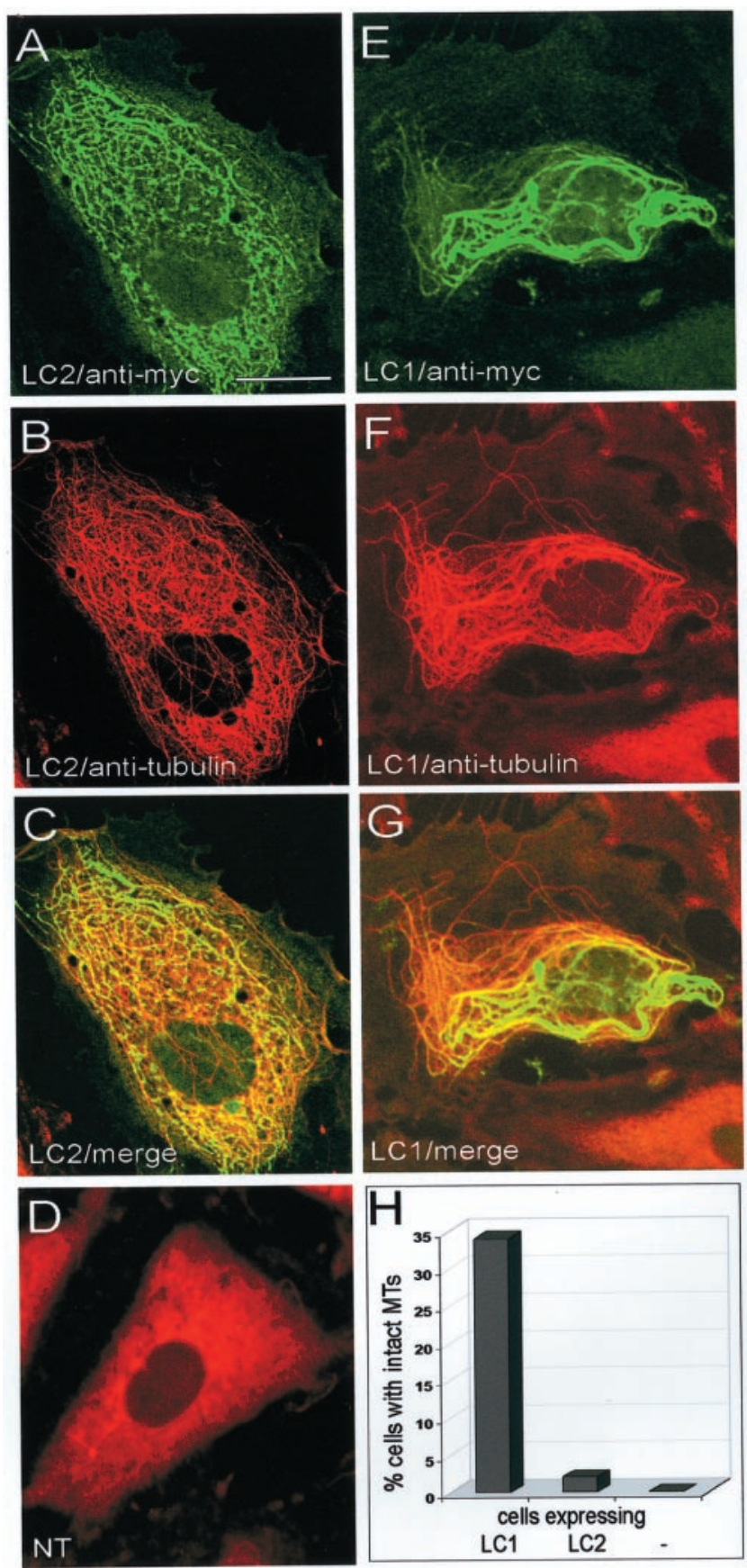

Figure 3. Effects of LC2 and LC1 on microtubule stability in cells treated with nocodazole. Nontransfected PtK2 cells $(N T, D)$ or PtK2 cells transfected with myc-tagged LC2 $(A-C)$ or myc-tagged LC1 $(E-G)$ were treated with nocodazole $(30-45 \mathrm{~min}, 10 \mu \mathrm{g} / \mathrm{ml})$ and then analyzed by double immunofluorescence microscopy using antibodies against tubulin $(B, D, F)$ and the myc-tag $(A, E)$. Microtubules were depolymerized in untransfected cells $(D)$, whereas intact microtubules were found in cells expressing LC2 $(B)$ and LC1 $(F)$. $C$ and $G$ represent merged confocal images of $A+B$ and $E+F$, respectively. Scale bar, $20 \mu \mathrm{m}$. $H$, One hundred randomly chosen transfected cells were assessed for the presence of intact microtubules (MTs). Quantitative analysis revealed that LC1 was considerably more efficient than LC2 in protecting microtubules against depolymerization (-, untransfected cells).

found (Fig. $3 A-C, E-G$ ), and in each case stable microtubules were decorated with the respective light chain, albeit at varying levels (Fig. 3C, $G$ ), again supporting the assumption that binding of the light chains is necessary for stabilization. However, quan- titative analysis revealed that the number of cells with stable microtubules was greatly diminished in LC2-expressing cells compared with LC1-expressing cells (Fig. $3 H$ ).

\section{The MAP1A light chain binds to microtubules via its $\mathrm{NH}_{2}$ terminus}

Amino acid sequence comparison of light chains LC1 and LC2 reveals a highly homologous region located at the $\mathrm{COOH}$ terminus, whereas no significant similarity can be found at the $\mathrm{NH}_{2}$ terminal domains (Langkopf et al., 1992). It has been shown previously by in vitro cosedimentation assays that the microtubule-binding domain of LC1 is located in the $\mathrm{NH}_{2}$-terminal half of the protein (Zauner et al., 1992). It was surprising to find that LC2, despite the lack of homology to the microtubulebinding domain of LC1, also bound to microtubules in vivo. To map the microtubule-binding domain of LC2, we performed microtubule cosedimentation assays using the full-length LC2 protein and deletion mutants (Fig. $4 A$ ). Full-length LC1 protein and $\mathrm{LC1}$ fragments comprising the $\mathrm{NH}_{2}$-terminal or $\mathrm{COOH}$ terminal domain were used as controls (Fig. 4A). After incubation with polymerized microtubules, only LC1 and LC2 proteins containing the $\mathrm{NH}_{2}$-terminal domain were found to cosediment to a significant extent (Fig. $4 B$ ). Absence of the $\mathrm{NH}_{2}$-terminal domains of LC1 and LC2 led to complete loss of microtubule binding. Thus, the COOH-terminal domains of LC1 and LC2 were found almost exclusively in the supernatant and therefore were not capable of mediating binding of the light chain proteins to microtubules. This result also showed that cosedimentation was specific and not merely attributable to unspecific binding of His-tag-containing proteins. In control experiments performed in the absence of microtubules, LC1 and LC2 proteins were recovered primarily in the supernatant. These results demonstrated the functional similarity of the $\mathrm{NH}_{2}$-terminal regions of $\mathrm{LC1}$ and LC2. Despite their structural diversity, the $\mathrm{NH}_{2}$-terminal domains of both proteins act as equally efficient microtubulebinding domains of the respective light chain.

\section{LC1 and LC2 proteins efficiently promote tubulin polymerization in vitro}

The ability of LC1 and LC2 proteins to induce the assembly of microtubules was investigated in reconstitution experiments. Previously, the influence of MAP1A and MAP1B on polymerization of microtubules in vitro has been monitored using the entire MAP1A and MAP1B heavy-chain/light-chain complex purified from brain (Pedrotti et al., 1993; Pedrotti and Islam, 1995). Here, we tested the influence of the individual light chains LC1 and LC2 on tubulin polymerization by turbidimetric time course experiments. Rapid polymerization of tubulin was induced by the fulllength light-chain proteins and deletion mutants comprising the $\mathrm{NH}_{2}$-terminal domains, each added at molar ratios of 1:40 to 1:1.5 to tubulin (Fig. 5). Microtubule formation appeared to proceed with little or no delay after addition of LC1 and LC2. Whereas polymerization induced by the full-length light chains commenced immediately, a lag phase preceded polymerization induced by the $\mathrm{NH}_{2}$-terminal fragments, resulting in a sigmoidal shape of the increase in absorbance over time. This lag phase was shortened but not abolished by an increase in the concentration of the light-chain fragment. Moreover, increasing the concentration of the $\mathrm{NH}_{2}$-terminal light-chain fragment led to an increase in the formation of microtubules, as evidenced by the higher plateau value of absorbance. The COOH-terminal domains of LC1 (Fig. 5) and LC2 (data not shown) added at a molar ratio of 1:1.5 to 

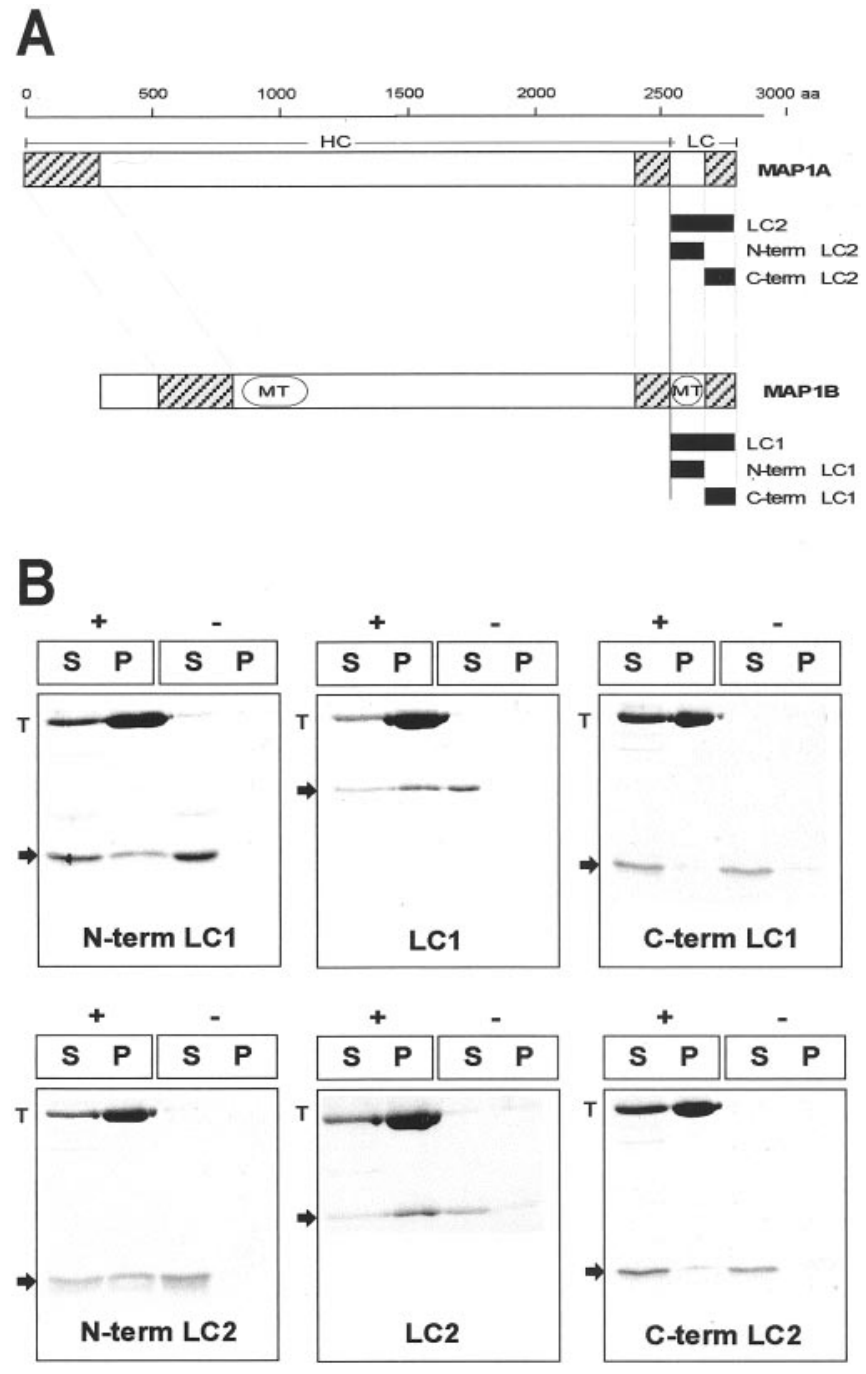

Figure 4. A, Schematic of MAP1A and MAP1B heavy and light chains ( $H C$ and $L C$, respectively) and cDNA constructs used in this study. The scale at the top displays amino acid $(a a)$ residue positions. Domains of sequence homology between MAP1A and MAP1B (hatched boxes) and the microtubule-binding domains $(M T)$ in the heavy and light chains of MAP1B are indicated. The cDNA constructs used are depicted as filled boxes. LC1, Full-length MAP1B light chain (amino acids 2210-2459); $N$-term LC1, amino acids 2210-2336; $C$-term LC1, amino acids 2335-2459; $L C 2$, full-length MAP1A light chain (amino acids 2554-2774); $N$-term $L C 2$, amino acids 2554-2659; C-term LC2, amino acids 2650-2774. For transfection studies, proteins were tagged with an $\mathrm{NH}_{2^{-}}$or $\mathrm{COOH}-$ terminal myc peptide. Proteins used for biochemical analysis were tagged with an $\mathrm{NH}_{2}$ - or $\mathrm{COOH}$-terminal 6xHis tag. $B, \mathrm{LC} 1$ and $\mathrm{LC} 2$ interact with microtubules in vitro. LC1 and LC2 proteins (arrows) were sedimented in the presence $(+)$ or absence $(-)$ of polymerized taxol-stabilized microtubules. Equal amounts of supernatant $(S)$ and pellet $(P)$ fractions were analyzed by SDS-PAGE and Coomassie blue staining. Proteins containing the $\mathrm{NH}_{2}$-terminal microtubule-binding domain of LC1 ( $N$-term LC1 and $L C 1$ ) and LC2 ( $N$-term $L C 2$ and $L C 2$ ) were found to cosediment with tubulin $(T)$, whereas only trace amounts of the $\mathrm{COOH}$-terminal domains of LC1 (C-term LC1) and LC2 (C-term LC2) were found in the pellet fraction.

tubulin did not induce microtubule polymerization above background levels. In fact, the $\mathrm{COOH}$-terminal domains served as negative controls and showed that the observed effects were specific and not attributable to the presence of the His tag. In the absence of light-chain proteins, little or no polymerization of tubulin was detected. Likewise, incubation of light-chain proteins
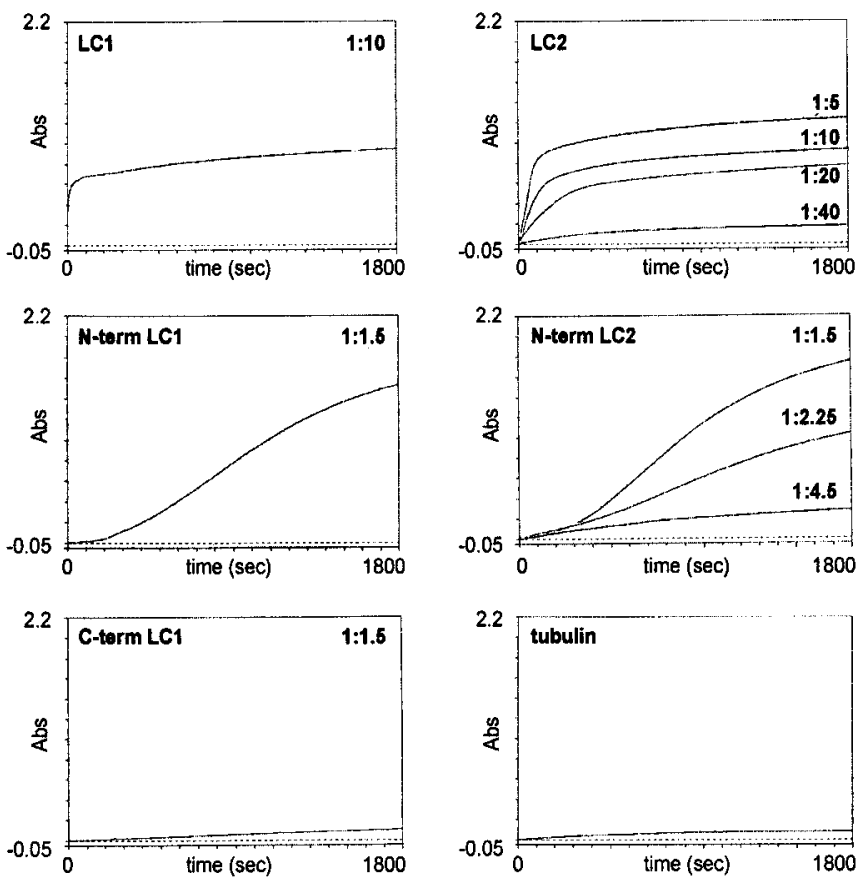

Figure 5. LC1 and LC2 proteins promote the polymerization of tubulin. LC1 and LC2 proteins were mixed with $1.5 \mathrm{mg} / \mathrm{ml}$ tubulin at molar ratios ranging from 1:1.5 to $1: 40$ as indicated. Polymerization of tubulin was started by placing the mixtures into prewarmed cuvettes and monitored by the change in absorbance at $350 \mathrm{~nm}$. Rapid polymerization of tubulin was observed using the full-length LC1 and LC2 proteins. Microtubule formation in the presence of $\mathrm{NH}_{2}$-terminal domains of LC1 ( $\mathrm{N}$-term LC1) and LC2 (N-term LC2) appeared to proceed with a delay; $\mathrm{COOH}$ terminal domains of LC1 (C-term LC1) and tubulin alone (tubulin) were used as controls. The dotted line in each panel defines absorbance level zero. Abs, Absorbance.

in the absence of tubulin did not lead to an increase of absorbance (data not shown). Taken together, these results demonstrate that the light chains of MAP1A and MAP1B have a profound influence on microtubule polymerization in vitro.

\section{The COOH-terminal domain of LC2 interacts with actin stress fibers in vivo}

Apart from its microtubule-binding activities, several studies have revealed that MAP1A interacts with microfilaments in vitro (Fujii et al., 1993; Pedrotti et al., 1994). However, these experiments had been conducted using the entire MAP1A heavy-chain/lightchain complex, and the localization of the actin-binding domain as well as experimental evidence obtained in vivo were not reported. Transfection of PtK2 cells with the full-length LC2 protein displayed colocalization with microtubules (Fig. $1 A-C$ ), and association with microfilaments was never observed. In contrast, transfection of the $\mathrm{LC} 2 \mathrm{COOH}$ terminus revealed that it is capable of binding to microfilaments. Cells expressing the LC2 $\mathrm{COOH}$ terminus displayed fibrillar staining and colocalization of the protein with actin stress fibers (Fig. 6). The latter was not affected by omission of the myc tag (data not shown). Staining with anti-myc antibodies also revealed a nuclear localization of this LC2 fragment, probably for reasons similar to those discussed above for full-length LC2.

\section{The COOH-terminal domains of LC1 and LC2 bind to actin in vitro}

We further tested whether binding of the corresponding homologous $\mathrm{COOH}$-terminal domains of LC1 and LC2 to actin stress 

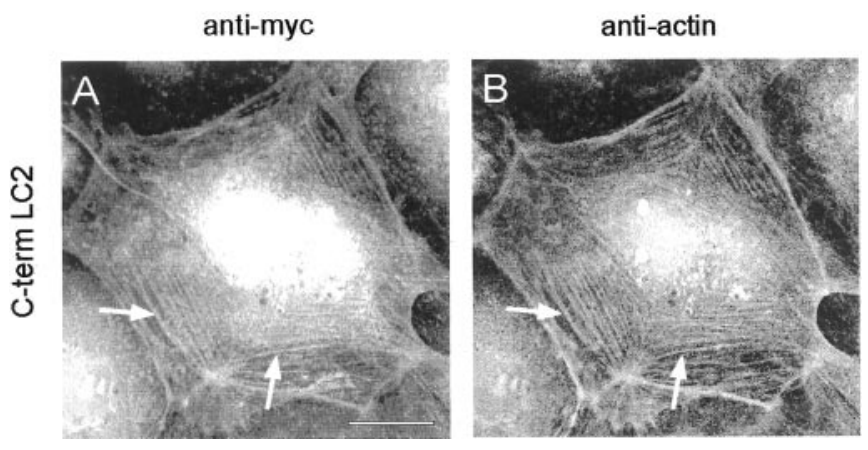

Figure 6. The $\mathrm{COOH}$-terminal domain of LC2 interacts with actin stress fibers. PtK2 cells were transfected with the $\mathrm{COOH}$-terminal domain of LC2. Double immunofluorescence microscopy using antibodies against the myc-tag $(A)$ and actin $(B)$ revealed colocalization of the myc-tagged LC2 COOH terminus with actin stress fibers ( $A$ and $B$, arrows). Scale bar, $10 \mu \mathrm{m}$.

fibers is attributable to a direct interaction with actin or whether it is mediated by an actin filament-associated protein. Purified recombinant full-length $\mathrm{LC} 1$ and LC2 proteins as well as deletion mutants comprising the respective $\mathrm{NH}_{2}$-terminal and $\mathrm{COOH}$ terminal domains were subjected to a solid-phase binding assay or to cosedimentation with actin filaments to investigate actinbinding properties in vitro. To perform solid-phase binding studies, microtiter plates were coated with $100 \mathrm{~nm}$ actin or BSA as a control. Coated wells were overlaid with increasing amounts of $\mathrm{Eu}^{3+}$-labeled LC1 and LC2, and bound protein was measured. For all proteins tested, binding to BSA was considered to be nonspecific and therefore was subtracted from the amount of protein bound to actin. Full-length LC1 and LC2 as well as their respective $\mathrm{COOH}$-terminal domain showed specific binding to actin, whereas fragments containing only the $\mathrm{NH}_{2}$ terminus of the respective light chain showed no binding (Fig. 7). At high concentrations, the full-length $\mathrm{LC} 1$ protein showed reduced binding compared with the $\mathrm{COOH}$-terminal domain (Fig. 7A). Concerning LC2, both proteins, the full-length LC2 and the COOHterminal domain, were approximately equally efficient in binding to actin (Fig. $7 B$ ).

To confirm these results by a second biochemical approach, we performed actin cosedimentation assays using the full-length LC1 and LC2 proteins and deletion mutants. Significant binding to actin was shown using the full-length LC1 and LC2 proteins (Fig. 8 ). In the absence of actin, both proteins were found predominantly in the supernatant, whereas incubation with actin led to complete sedimentation of the light-chain proteins. Sedimentation of F-actin remained unaffected in the presence of LC1 and LC2, indicating that neither protein prevented actin polymerization like F-actin capping or severing proteins (Matsudaira, 1992). Sedimentation of polymerized actin alone was almost complete, resulting in at least $90 \%$ of the protein being found in the pellet fraction (data not shown). Under the conditions of this assay, COOH-terminal domains of LC1 and LC2 pelleted in the absence of actin and therefore were not included in this assay. As expected, the $\mathrm{NH}_{2}$-terminal domains of LC1 and LC2 in the absence as well as the presence of actin were found almost exclusively in the supernatant. Thus, in cosedimentation as well as $\mathrm{Eu}^{3+}$-overlay assays, we can exclude nonspecific binding attributable to His tags. These results confirmed and extended our results obtained in vivo (Fig. 6) and demonstrated by two independent in vitro assays that the light chains of MAP1A and
A

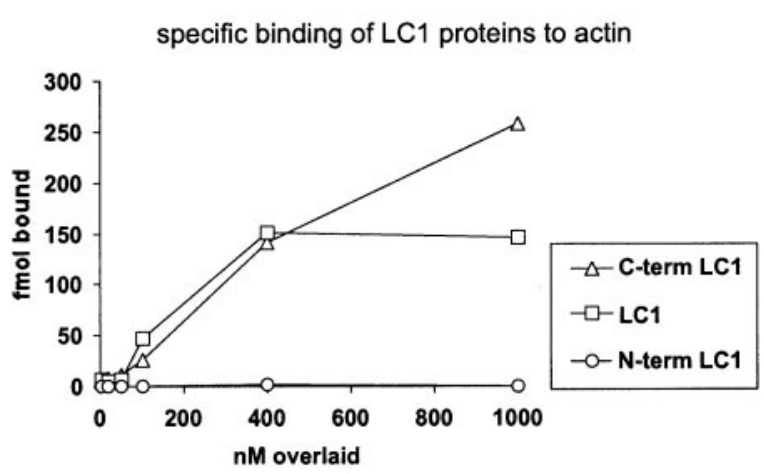

B

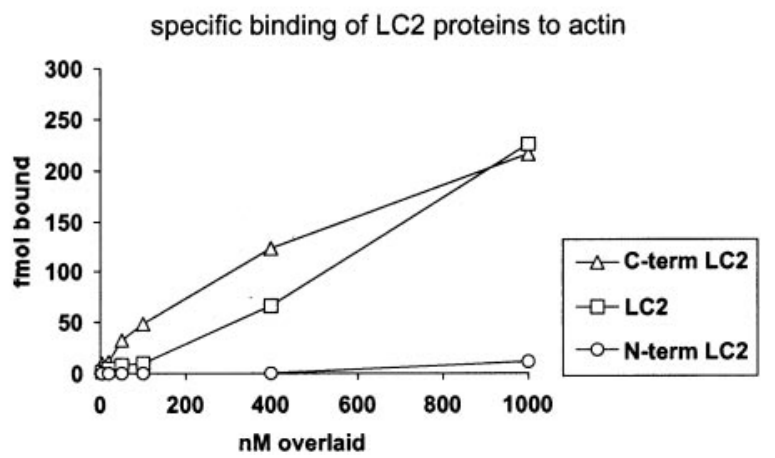

Figure 7. The COOH-terminal domains of LC1 and LC2 interact with actin in vitro. Microtiter plates coated with $100 \mathrm{~nm}$ actin were overlaid with various concentrations of $\mathrm{Eu}^{3+}$-labeled LC1 $(A)$ and LC2 $(B)$ proteins. Full-length LC1 and LC2 as well as their respective $\mathrm{COOH}$-terminal domains (C-term LC1 and C-term LC2) showed specific binding to actin. No binding was observed using the $\mathrm{NH}_{2}$-terminal domains of $\mathrm{LC1}(\mathrm{N}$ term LC1) and LC2 (N-term LC2). The amounts of protein bound represent the results of three measurements of at least two independent experiments.

MAP1B can directly interact with actin through an actin-binding site located in the homologous $\mathrm{COOH}$-terminal domains.

\section{DISCUSSION}

It is generally accepted that the demands on the neuronal cytoskeleton change after transition from the developmental state to the mature state. The switch from MAP1B to MAP1A during postnatal development might be part of the mechanism to adapt the cytoskeleton to these changing requirements. If this were the case, one would expect distinct effects of MAP1A and MAP1B on cellular microtubules and microfilaments. However, previous studies using the entire protein complex, consisting of heavy and light chains, revealed only moderate and similar effects on microtubule shape and stability (Noble et al., 1989; Takemura et al., 1992; Vaillant et al., 1998). To elucidate the functional properties of MAP1A and to successfully unravel the existence of MAP1Aspecific features that might be instrumental in the switch from the developmental to the mature cytoskeleton, we chose to investigate the functional properties of the light chain of MAP1A, LC2, and to compare them with properties of LC1. Our findings demonstrate that (1) MAP1A and MAP1B light chains indeed have specific effects on microtubules, (2) both proteins are potential linkers of microtubules and microfilaments, and (3) the properties of MAP1A and MAP1B are at least in part attributable to distinct activities of their light chains. 

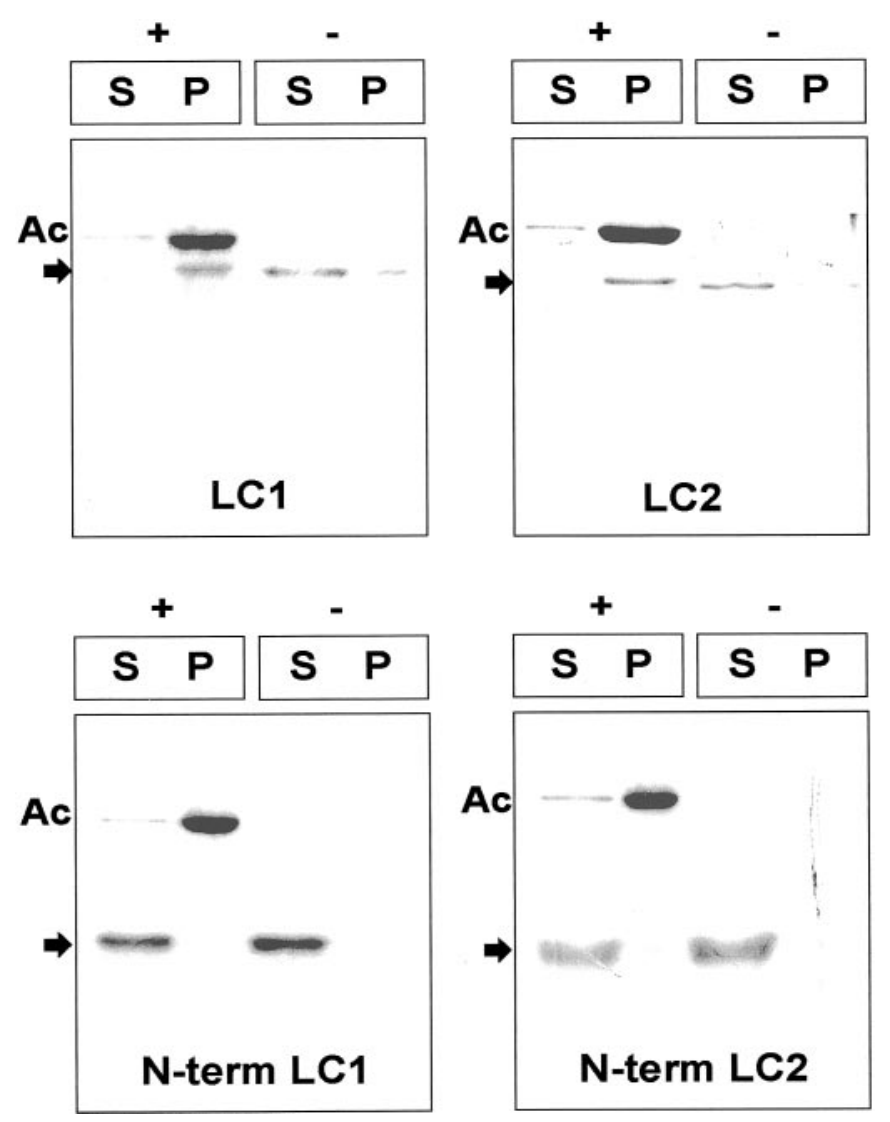

Figure 8. LC1 and LC2 cosediment with actin $(A c)$ via their $\mathrm{COOH}-$ terminal domain. LC1 and LC2 proteins (arrows) were sedimented in the presence $(+)$ or absence $(-)$ of polymerized actin. Equal amounts of supernatant $(S)$ and pellet $(P)$ fractions were analyzed by SDS-PAGE and Coomassie blue staining. Full-length LC1 and LC2 were found to cosediment with actin, whereas only trace amounts of the $\mathrm{NH}_{2}$-terminal domains of LC1 ( $N$-term LC1) and LC2 ( $N$-term LC2) were found in the pellet fraction.

Ectopic expression of LC2 in PtK2 cells revealed binding of the protein to microtubules. Previously, two separate regions located on the MAP1A heavy chain had been implicated in microtubule binding. Cravchik et al. (1994) reported results of colocalization studies indicating that the microtubule-binding domain of MAP1A was located on the heavy chain between amino acids 1300 and 1600. Conversely, Vaillant et al. (1998) concluded from their transfection experiments using a variety of MAP1A deletion constructs that amino acids 281-336 and/or amino acids 540-630 of MAP1A are implicated in microtubule binding. These authors also demonstrated that full-length MAP1A consisting of heavy and light chains can bind to microtubules, but the existence of a microtubule-binding domain in LC2 had not been reported. We demonstrate here that $\mathrm{LC} 2$ by itself not only binds to cellular microtubules but also stabilizes them against the destructive effects of colchicine. Binding of LC2 was confirmed in vitro, and we have shown that LC2 has profound effects on microtubule polymerization. The $\mathrm{NH}_{2}$-terminal domain of LC2 was necessary and sufficient for microtubule binding and induction of polymerization. Nonetheless, microtubule formation was more rapid in the presence of the full-length LC2 protein comprising $\mathrm{NH}_{2}$-and $\mathrm{COOH}$-terminal domains than the $\mathrm{NH}_{2}-$ terminal domain alone. Similar results were obtained for LC1. Because the $\mathrm{COOH}$-terminal domains of either light chain did not themselves interact with microtubules, their enhancement of microtubule formation when present as part of the full-length light chain must be caused by a different mechanism. The $\mathrm{COOH}$ terminal domains of both light chains share a high degree of sequence identity. We have shown previously that LC1 dimerizes or even oligomerizes via this $\mathrm{COOH}$-terminal domain (Tögel et al., 1998b). It is tempting to speculate that dimerization/oligomerization of the light chains might have an influence on microtubule polymerization. Association of light chains via their $\mathrm{COOH}$-terminal domains could facilitate microtubule polymerization by simply recruiting more tubulin subunits, which would accelerate the nucleation event preceding microtubule elongation. This would in turn account for the significantly shortened lag phase we observed using the full-length light chains. In agreement with this hypothesis, such influence on the nucleation of microtubule assembly has been described previously using the entire MAP1B protein (Vandecandelaere et al., 1996). Rapid polymerization of microtubules has also been observed using the entire MAP1A and MAP1B protein complex purified from brain (Pedrotti et al., 1993; Pedrotti and Islam, 1995). At similar stoichiometric amounts of protein, the polymerization monitored in these studies resembles the results we obtained by solely using the light chains, suggesting that microtubule polymerization is enhanced by the light chains, leaving the heavy chains rather dispensable.

While the LC2 $\mathrm{NH}_{2}$ terminus harbors a domain that is necessary and sufficient for microtubule binding and polymerization, its $\mathrm{COOH}$-terminal half contains an actin-binding domain. Ectopic expression of the $\mathrm{LC} 2 \mathrm{COOH}$ terminus revealed interaction with actin stress fibers. Moreover, the ability of LC2 to interact with actin via its $\mathrm{COOH}$-terminal domain in solid-phase binding studies and cosedimentation assays suggests a direct association with actin. Similar results were obtained for $\mathrm{LC} 1$ and its $\mathrm{COOH}$ terminus. Previous observations of the interaction of the entire MAP1A complex with microfilaments (Fujii et al., 1993; Pedrotti et al., 1994) can thus be explained by the actin-binding properties of the light chain demonstrated here. An interesting observation in our studies was that full-length recombinant LC1 and LC2, synthesized in and purified from $E$. coli, were able to bind to microtubules and actin in vitro, whereas LC1 and LC2 ectopically expressed in PtK2 cells associated exclusively with microtubules, although their $\mathrm{COOH}$-terminal domains can bind to actin in vitro and in vivo. It is conceivable that actin binding of full-length light chains is inhibited or regulated by post-translational modifications such as phosphorylation, which could take place in PtK2 cells but not in E. coli. Indeed, it has been demonstrated that binding of the MAP1B heavy-chain/light-chain complex to actin requires previous treatment with alkaline phosphatase (Pedrotti and Islam, 1996), and that phosphorylation of MAP1B enhances binding to microtubules (Brugg and Matus, 1988; Diaz-Nido et al., 1988; Ulloa et al., 1993). A similar mechanism might operate for MAP1A and LC2.

Through our comparison of LC1 and LC2, we discovered that the two light chains do indeed have similar but distinct activities, consistent with the notion that the switch from LC1 to LC2 during postnatal brain development could reflect the changing demands on the neuronal cytoskeleton. Both LC1 and LC2 each have an $\mathrm{NH}_{2}$-terminal microtubule-binding domain linked to a $\mathrm{COOH}$ terminal actin-binding domain. Both light chains bind to microtubules and actin in vivo and in vitro and enhance microtubule polymerization in vitro. The major differences are in the effects on cellular microtubules. Whereas LC1 induces the formation of 
microtubule bundles and loops reminiscent of microtubules in advancing growth cones (Tsui et al., 1984; Sabry et al., 1991; Tanaka and Kirschner, 1991; Challacombe et al., 1996; Dent et al., 1999), LC2 does not appear to change microtubule arrangements. In addition, LC2 stabilizes microtubules only against colchicine, but not nocodazole, whereas LC1 is effective against both drugs. Colchicine binds to tubulin through a bipartite binding site consisting of $\mathrm{A}$ and $\mathrm{C}$ subsites, of which presumably only the $\mathrm{A}$ site is used by nocodazole (Wilson and Jordan, 1994). The differential effects of LC1 and LC2 might be caused by interference with the $\mathrm{A}$ and $\mathrm{C}$ site, respectively. Thus, both could prevent colchicine binding by blocking either the $\mathrm{A}$ or the $\mathrm{C}$ site, whereas only LC1 would also block nocodazole binding. These differences in light-chain action on microtubules can be attributed to the differences in primary structure of the respective microtubulebinding domains located in the $\mathrm{NH}_{2}$-terminal half of the two light chains, because these are the only parts of the light chains that are not homologous in sequence. Thus, the effect of the switch from LC1 to LC2 during postnatal development is in essence the replacement of the LC1 microtubule-binding domain by the corresponding LC2 domain with a different effect on microtubules.

The present study demonstrates the importance of the light chains for MAP1B and MAP1A function. In the brain, the light chains can be found in two settings. One fraction of LC1 and LC2 is found associated with the corresponding heavy chains (Schoenfeld et al., 1989). We have previously obtained evidence that in the MAP1B heavy-chain/light-chain complex, the heavy chain acts as a regulatory subunit (Tögel et al., 1998b). Our present findings of microtubule- and actin-binding activities of LC2 raise the possibility that in the MAP1A complex as well, the light chain is the active subunit and the heavy chain acts as a regulator. Of particular interest in this context is the fact that heterologous complexes can be detected in which LC1 is bound to a MAP1A heavy chain (Schoenfeld et al., 1989). Thus, it appears that during postnatal development, in addition to the switch from MAP1B to MAP1A, LC1 can participate in a mixed complex consisting of the active subunit LC1 with its distinct "developmental" effects on cellular microtubules and the putative "adult" regulator, the heavy chain of MAP1A. Whatever the true function of the MAP1A heavy chain may be, the documented light-chain replacement on a given heavy chain at a certain transition period during development is consistent with the above-mentioned model of light- and heavy-chain duties in the MAP1 complex and emphasizes the importance of the light chains.

A second and larger fraction of the light chains is found not to be associated with heavy chains. This has been demonstrated for LC1 (Mei et al., 2000b) and could be true for LC2 as well. The results presented here identify which activities such uncomplexed light chains have. We propose that the microtubule- and actinbinding activities reported here will be the key to the functions of the light chains.

\section{REFERENCES}

Asai DJ, Thompson WC, Wilson L, Dresden CF, Schulman H, Purich DL (1985) Microtubule-associated proteins (MAPs): a monoclonal antibody to MAP1 decorates microtubules in vitro but stains stress fibers and not microtubules in vivo. Proc Natl Acad Sci USA 82:1434-1438.

Binder LI, Frankfurter A, Kim H, Caceres A, Payne MR, Rebhun LI (1984) Heterogeneity of microtubule-associated protein 2 during rat brain development. Proc Natl Acad Sci USA 81:5613-5617.

Bradford MM (1976) A rapid and sensitive method for the quantitation of microgram quantities of protein utilizing the principle of protein-dye binding. Anal Biochem 72:248-254.

Brugg B, Matus A (1988) PC12 cells express juvenile microtubule- associated proteins during nerve growth factor-induced neurite outgrowth. J Cell Biol 107:643-650.

Brugg B, Reddy D, Matus A (1993) Attenuation of microtubuleassociated protein 1B expression by antisense oligonucleotides inhibits initiation of neurite outgrowth. Neuroscience 52:489-496.

Calvert R, Anderton BH (1985) A microtubule-associated protein (MAP1) which is expressed at elevated levels during development of the rat cerebellum. EMBO J 4:1171-1176.

Challacombe JF, Snow DM, Letourneau PC (1996) Actin filament bundles are required for microtubule reorientation during growth cone turning to avoid an inhibitory guidance cue. J Cell Sci 109:2031-2040.

Cravchik A, Matus A (1993) A novel strategy for the immunological tagging of cDNA constructs. Gene 137:139-143.

Cravchik A, Reddy D, Matus A (1994) Identification of a novel microtubule-binding domain in microtubule-associated protein $1 \mathrm{~A}$ (MAP1A). J Cell Sci 107:661-672.

Dent EW, Callaway JL, Szebenyi G, Baas PW, Kalil K (1999) Reorganization and movement of microtubules in axonal growth cones and developing interstitial branches. J Neurosci 19:8894-8908.

Diaz-Nido J, Serrano L, Mendez E, Avila J (1988) A casein kinase II-related activity is involved in phosphorylation of microtubuleassociated protein MAP-1B during neuroblastoma cell differentiation. J Cell Biol 106:2057-2065.

DiTella MC, Feiguin F, Carri N, Kosik KS, Cáceres A (1996) MAP-1B/ TAU functional redundancy during laminin-enhanced axonal growth. J Cell Sci 109:467-477.

Edelmann W, Zervas M, Costello P, Roback L, Fischer I, Hammarback JA, Cowan N, Davies P, Wainer B, Kucherlapati R (1996) Neuronal abnormalities in microtubule-associated protein $1 \mathrm{~B}$ mutant mice. Proc Natl Acad Sci USA 93:1270-1275.

Feick P, Foisner R, Wiche G (1991) Immunolocalization and molecular properties of a high molecular weight microtubule-bundling protein (syncolin) from chicken erythrocytes. J Cell Biol 112:689-699.

Fujii T, Watanabe M, Ogoma Y, Kondo Y, Arai T (1993) Microtubuleassociated proteins, MAP1A and MAP1B, interact with F-actin in vitro. J Biochem 114:827-829.

Garner CC, Garner A, Huber G, Kozak C, Matus A (1990) Molecular cloning of microtubule-associated protein 1 (MAP1A) and microtubule-associated protein 5 (MAP1B): identification of distinct genes and their differential expression in developing brain. J Neurochem 55:146-154.

González-Billault C, Demandt E, Wandosell F, Torres M, Bonaldo P, Stoykova A, Chowdhury K, Gruss P, Avila J, Sánchez MP (2000) Perinatal lethality of microtubule-associated protein 1B-deficient mice expressing alternative isoforms of the protein at low levels. Mol Cell Neurosci 16:408-421.

Gonzalez-Billault C, Avila J, Caceres A (2001) Evidence for the role of MAP1B in axon formation. Mol Biol Cell 12:2087-2098.

Gossen M, Bujard H (1992) Tight control of gene expression in mammalian cells by tetracycline-responsive promoters. Proc Natl Acad Sci USA 89:5547-5551.

Hammarback JA, Obar RA, Hughes SM, Vallee RB (1991) MAP1B is encoded as a polyprotein that is processed to form a complex N-terminal microtubule-binding domain. Neuron 7:129-139.

Karr TL, White HD, Purich DL (1979) Characterization of brain microtubule proteins prepared by selective removal of mitochondrial and synaptosomal components. J Biol Chem 254:6107-6111.

Langkopf A, Hammarback JA, Müller R, Vallee RB, Garner CC (1992) Microtubule-associated proteins 1A and LC2. J Biol Chem 267:1656116566.

Lewis SA, Sherline P, Cowan NJ (1986) A cloned cDNA encoding MAP1 detects a single copy gene in mouse and a brain-abundant RNA whose level decreases during development. J Cell Biol 102:2106-2114.

Matsudaira P (1992) Mapping structural and functional domains in actin-binding proteins. In: The cytoskeleton: a practical approach (Carraway K, Carraway C, eds), pp 73-98. Oxford: Oxford UP.

Mei X, Sweatt AJ, Hammarback JA (2000a) Microtubule-associated protein 1 subunit expression in primary cultures of rat brain. Brain Res Bull 53:801-806.

Mei X, Sweatt AJ, Hammarback JA (2000b) Regulation of microtubuleassociated protein 1B (MAP1B) subunit composition. J Neurosci Res 62:56-64.

Meixner A, Haverkamp S, Wässle H, Führer S, Thalhammer J, Kropf N, Bittner RE, Lassmann H, Wiche G, Propst F (2000) MAP1B is required for axon guidance and is involved in the development of the central and peripheral nervous system. J Cell Biol 151:1169-1178.

Noble M, Lewis SA, Cowan NJ (1989) The microtubule binding domain of microtubule-associated protein MAP1B contains a repeated sequence motif unrelated to that of MAP2 and tau. J Cell Biol 109:3367-3376.

Pedrotti B, Islam K (1995) Microtubule associated protein 1B (MAP1B) promotes efficient tubulin polymerisation in vitro. FEBS Lett 371:29-31.

Pedrotti B, Islam K (1996) Dephosphorylated but not phosphorylated 
microtubule associated protein MAP1B binds to microfilaments. FEBS Lett 388:131-133.

Pedrotti B, Soffientini A, Islam K (1993) Sulphonate buffers affect the recovery of microtubule-associated proteins MAP1 and MAP2: evidence that MAP1A promotes microtubule assembly. Cell Motil Cytoskeleton 25:234-242.

Pedrotti B, Colombo R, Islam K (1994) Microtubule associated protein MAP1A is an actin-binding and crosslinking protein. Cell Motil Cytoskeleton 29:110-116.

Riederer B, Cohen R, Matus A (1986) MAP5: a novel brain microtubule-associated protein under strong developmental regulation. J Neurocytol 15:763-775.

Sabry JH, O'Connor TP, Evans L, Toroian-Raymond A, Kirschner M, Bentley D (1991) Microtubule behavior during guidance of pioneer neuron growth cones in situ. J Cell Biol 115:381-395.

Safaei R, Fischer I (1989) Cloning of a cDNA encoding MAP1B in rat brain: regulation of mRNA levels during development. J Neurochem 52:1871-1879.

Schoenfeld TA, Obar RA (1994) Diverse distribution and function of fibrous microtubule-associated proteins in the nervous system. Int Rev Cytol 151:67-137.

Schoenfeld TA, McKerracher L, Obar R, Vallee RB (1989) MAP 1A and MAP $1 \mathrm{~B}$ are structurally related microtubule associated proteins with distinct developmental patterns in the CNS. J Neurosci 9:1712-1730.

Steinböck FA, Nikolic B, Coulombe PA, Fuchs E, Traub P, Wiche G (2000) Dose-dependent linkage, assembly inhibition and disassembly of vimentin and cytokeratin 5/14 filaments through plectin's intermediate filament-binding domain. J Cell Sci 113:483-491.

Takei Y, Kondo S, Harada A, Inomata S, Noda T, Hirokawa N (1997) Delayed development of nervous system in mice homozygous for disrupted microtubule-associated protein 1B (MAP1B) gene. J Cell Biol 137:1615-1626.

Takemura R, Okabe S, Umeyama T, Kanai Y, Cowan NJ, Hirokawa N (1992) Increased microtubule stability and alpha tubulin acetylation in cells transfected with microtubule-associated proteins MAP1B, MAP2 or tau. J Cell Sci 103:953-964.

Tanaka EM, Kirschner MW (1991) Microtubule behavior in the growth cones of living neurons during axon elongation. $\mathrm{J}$ Cell Biol 115:345-363.

Tögel M, Wiche G, Propst F (1998a) Evidence against structural and functional identity of microtubule-associated protein MAP1B and proteoglycan claustrin. FEBS Lett 423:254-258.

Tögel M, Wiche G, Propst F (1998b) Novel features of the light chain of microtubule-associated protein MAP1B: microtubule stabilization, self interaction, actin filament binding, and regulation by the heavy chain. J Cell Biol 143:695-707.

Tsui HT, Lankford KL, Ris H, Klein WL (1984) Novel organization of microtubules in cultured central nervous system neurons: formation of hairpin loops at ends of maturing neurites. J Neurosci 4:3002-3013.

Ulloa L, Díaz-Nido J, Avila J (1993) Depletion of casein kinase II by antisense oligonucleotide prevents neuritogenesis in neuroblastoma cells. EMBO J 12:1633-1640.

Vaillant AR, Müller R, Langkopf A, Brown DL (1998) Characterization of the microtubule-binding domain of microtubule-associated protein $1 \mathrm{~A}$ and its effects on microtubule dynamics. J Biol Chem 273:13973-13981.

Vandecandelaere A, Pedrotti B, Utton MA, Calvert RA, Bayley PM (1996) Differences in the regulation of microtubule dynamics by microtubule-associated protein MAP1B and MAP2. Cell Motil Cytoskeleton 35:134-146.

Weinert T, Cappuccinelli P, Wiche G (1982) Potent microtubule inhibitor protein from Dictyostelium discoideum. Biochemistry 21:782-789.

Wiche G, Oberkanins C, Himmler A (1991) Molecular structure and function of microtubule-associated proteins. Int Rev Cytol 124:217-273.

Wilson L, Jordan MA (1994) Pharmacological probes of microtubule function. In: Microtubules (Hyams J, Lloyd C, eds), pp 59-83. New York: Wiley.

Zauner W, Kratz J, Staunton J, Feick P, Wiche G (1992) Identification of two distinct microtubule binding domains on recombinant rat MAP 1B. Eur J Cell Biol 57:66-74. 